\title{
Neurofilaments and tau proteins in cerebrospinal fluid and serum in dementias and neuroinflammation
}

\author{
Lenka Fialova ${ }^{a *}$, Ales Bartos ${ }^{b, c *}$, Jana Svarcova ${ }^{d}$
}

\begin{abstract}
Aims. The aim of this study was to evaluate the diagnostic potential of cerebrospinal fluid (CSF) and serum levels of neurocytoskeletal proteins and their ratios for the diagnosis of dementias and to assess the differences in neurocytoskeletal proteins between neurodegeneration and neuroinflammation.

Methods. CSF and serum levels of neurofilament light subunits (NFL) and neurofilament heavy subunits (NFH) as well as CSF levels of total tau ( $t$-tau) and phosphorylated tau ( $p$-tau) proteins were determined using ELISA in 20 Alzheimer's disease patients (AD group), 13 patients with other dementias (OD group), 17 patients with inflammatory aseptic neuro-infections (IP), and 20 aged-matched cognitively normal elderly persons (NC group).

Results. The ratio CSF p-tau/t-tau was significantly higher in the NC group than that in the AD or OD groups $(P<0.0005$ for each group). The CSF NFH/p-tau and CSF NFL/p-tau ratios were significantly lower in AD patients than OD patients $(P \leq 0.003)$. Serum and CSF NFL and CSF NFH levels were significantly higher in OD patients than AD patients $(P \leq 0.03)$. The lowest values of the CSF NFL/NFH ratio were found in the IP group and they significantly differed from those in normal controls $(P<0.0001)$ and any dementia group (IP vs. AD $P<0.0001$; IP vs. OD $P=0.03)$.

Conclusion. CSF tau proteins and their index differentiated between AD or OD patients and cognitively normal subjects, while CSF levels of neurofilaments expressed as their index seem to contribute to the discrimination between patients with neuroinflammation and normal controls or AD patients.
\end{abstract}

Key words: Alzheimer's disease, dementias, cytoskeletal proteins, neurofilaments, neuroinflammation, tau protein, cerebrospinal fluid, serum

Received: June 26, 2017; Accepted with revision: September 4, 2017; Available online: September 25, 2017 https://doi.org/10.5507/bp.2017.038

aInstitute of Medical Biochemistry and Laboratory Diagnostics, First Faculty of Medicine, Charles University and General University Hospital in Prague, Katerinska 32, 12108 Prague, Czech Republic

${ }^{b}$ National Institute of Mental Health, Topolova 748, 25067 Klecany, Czech Republic

'Department of Neurology, Third Faculty of Medicine, Charles University and University Hospital Kralovske Vinohrady, Srobarova 50, 100 34 Prague, Czech Republic

${ }^{d}$ Department of Medical Chemistry and Clinical Biochemistry, Motol University Hospital, Prague, Czech Republic

Corresponding author: Lenka Fialova, e-mail: Ifial@If1.cuni.cz

*These authors contributed equally to this paper

\section{INTRODUCTION}

Alzheimer's disease $(\mathrm{AD})$ is characterized by specific neuropathological features involving the formation of beta-amyloid plaques and the presence of neurofibrillary tangles (NFT) in the brain ${ }^{1,2}$. Extracellularly located betaamyloid plaques contain mainly insoluble peptide betaamyloid (Abeta), while neurofibrillary tangles located intraneuronally are composed predominantly of cytoskeletal protein hyperphosphorylated tau ${ }^{2,3}$. The presence of beta-amyloid plaques and NFT are reflected in the altered levels of beta-amyloid, phosphorylated tau protein (p-tau) and total tau protein (t-tau) in CSF (further referred to as a CSF triplet), and less specific processes like axonal damage by altered neurofilament (NF) levels ${ }^{4-6}$. Determination of these proteins facilitates differentiation of patients with $\mathrm{AD}$ from cognitively normal elderly individuals and other dementias ${ }^{7-9}$.

In addition to the CSF triplet (t-tau, p-tau, Abeta proteins), NFL in CSF is also considered as a marker of neurocytoskeleton impairment in AD. Generally, neurofilament light subunits (NFL) are considered to be a biomarker of large-caliber myelinated axon injury ${ }^{10}$. Moreover, detailed analysis showed that NFT contain not only p-tau but also other phosphorylated proteins including neurofilaments ${ }^{11}$. A comparison between nine cohorts with $\mathrm{AD}$ patients and eight control cohorts revealed 2.35 times average NFL elevation in $\mathrm{AD}$ ( ref. $^{9}$ ). Correlation of CSF NFL with accelerated cognitive decline, changes in white matter and increased brain atrophy is found in patients with mild cognitive impairment ${ }^{12}$. Recently, elevated NFL levels were also described in the serum/plasma of AD patients ${ }^{13-15}$. NFH elevation in CSF seems to be less prominent in AD. Significantly higher CSF NFH levels as well as non-significant elevation of CSF NFH in AD patients have been described ${ }^{16-18}$. However, higher NF levels in CSF have been found in other dementias, and hence determination of NFL and NFH may be valuable in the differentiation of dementias ${ }^{5,19,20}$.

In our study we focused on neurocytoskeletal proteins as biomarkers. Measurements of well-established CSF total and phosphorylated tau proteins were extended with 
a promising biomarker of NF ( ref. $^{9}$ ). Our complex evaluation included light and heavy subunits of NF not only in CSF, but we also tried to determine serum concentrations. Only a limited number of papers have reported on NF levels in the serum of dementia patients. The relationship between individual proteins expressed as ratios might also contribute to differential diagnosis of demen$\operatorname{tias}^{20,21}$. We assumed simultaneous elevation of selected cytoskeletal proteins released from damaged neurons or involved in NFT and other pathology in neurodegenerative dementias. Patients with AD and other dementias were compared not only with age-matched cognitively normal elderly controls but also with patients affected by neuro-infections. Neuroinflammation may result in different consequences compared to neurodegeneration in terms of concentrations or ratios of neuron-specific cytoskeletal proteins.

\section{MATERIALS AND METHODS}

\section{Participants}

We enrolled 70 individuals at the Department of Neurology or Memory Clinic of the University Hospital, Charles University, Prague. Participants were divided into four groups: (1) patients with AD (AD group; $n=20$ ); (2) patients with other dementias (OD group; $n=13$ ); (3) patients with inflammatory aseptic neuro-infections (IP group; $n=17$ ); and (4) cognitively normal elderly persons (NC group; $\mathrm{n}=20$ ) (Table 1).

The cognitive function of participants was evaluated using an updated Czech version of the Addenbrooke's Cognitive Examination-Revised (ACE-R-CZ). The test ACE-R-CZ is a $20-30$ min more detailed examination of cognitive functions than the Mini-Mental State Examination (MMSE) (ref. ${ }^{22-28}$ ). MMSE scores were derived from the ACE-R-CZ. Brain magnetic resonance imaging (MRI) or computer tomography imaging (when not suitable for MRI) were performed in all dementia patients and in the majority of participants. Intracranial pathology was excluded and hippocampal size was assessed using mediotemporal atrophy scores ${ }^{29,30}$.
The AD patients met a probable diagnosis of $\mathrm{AD}$ according to the NIA-AA (National Institute on AgingAlzheimer's Association) criteria by an experienced neurologist ${ }^{31}$. The patients with other dementias were carefully selected for this study. Most of them had autopsy confirmation. Four patients had frontotemporal lobar degeneration (FTLD) (three were autopsy-confirmed, one had amyotrophic lateral sclerosis as well). All other patients had autopsy-confirmed diagnoses - three with Creutzfeldt-Jacob diseases, one with corticobasal degeneration and one with Wernicke encephalopathy. The IP group consisted of patients with aseptic neuro-infections, mainly neuroborreliosis or herpetic infections. Normal elderly controls were age-matched with other groups. They were diagnosed as non-inflammatory conditions, mostly with polyneuropathy $(n=10)$; the rest included a variety of diseases (e.g. headache, Bell's palsy). They had normal MMSE and ACE-R-CZ scores and a normal CSF triplet (t-tau, p-tau, Abeta proteins) based on cut-offs established in our and other previous studies ${ }^{24,28,32,33}$. These patients underwent a lumbar puncture as part of a routine diagnostic work-up.

Lumbar CSF and paired blood samples were collected, centrifuged, aliquoted in $1 \mathrm{~mL}$ polypropylene tubes and stored on average within $1.5 \mathrm{~h}$ of sampling at $-80{ }^{\circ} \mathrm{C}$ in accordance with established guidelines until analysis ${ }^{34}$. The specimens were thawed just prior to the measurements.

Participants signed an informed consent. The research was approved by the Ethics Committee of the University Hospital Kralovske Vinohrady.

\section{Determination of neurocytoskeletal proteins and albumin}

All of the neurocytoskeletal proteins and Abeta peptides were measured by commercially available ELISA kits. The ELISAs were performed according to the manufacturer's instructions.

We used NF-Light ${ }^{\circledR}$ Neurofilament ELISA RUO (reseach use only) kits (UmanDiagnostics, Umeå, Sweden) and Phosphorylated Neurofilament H ELISA kits (pNFH) (BioVendor, Czech Republic) for the determination of light and heavy subunits of neurofilaments in

Table 1. Demographic and cognitive characteristics of the subjects.

\begin{tabular}{|c|c|c|c|c|}
\hline & $\begin{array}{c}\text { Alzheimer's } \\
\text { disease patients } \\
(n=20)\end{array}$ & $\begin{array}{l}\text { Other dementia } \\
\text { patients } \\
(n=13)\end{array}$ & $\begin{array}{c}\text { Patients with } \\
\text { neuroinflammation } \\
(n=17)\end{array}$ & $\begin{array}{c}\text { Normal patient } \\
\text { controls } \\
(n=20)\end{array}$ \\
\hline Female sex number (\%) & $17(85 \%)$ & $4(23 \%)$ & $10(58 \%)$ & $9(45 \%)$ \\
\hline Education (years) & $12 \pm 2$ & $15 \pm 4$ & $14 \pm 4$ & $13 \pm 2$ \\
\hline Age at sampling (years) & $71 \pm 7$ & $64 \pm 8$ & $70 \pm 8$ & $67 \pm 7$ \\
\hline MMSE score (0-30 points) & $22 \pm 3 *$ & $20 \pm 6 *$ & $28 \pm 4$ & $29 \pm 1$ \\
\hline ACE-R-CZ score (0-100 points) & $69 \pm 12 \dagger+$ & $66 \pm 22 \dagger^{\circ}$ & $86 \pm 12$ & $92 \pm 5$ \\
\hline
\end{tabular}

MMSE - the Mini-Mental State Examination; ACE-R-CZ - the updated Czech version of the Addenbrooke's Cognitive Examination-Revised

Data are expressed as mean \pm SD or number (\%).

$* P<0.0001$ vs. normal patients control and patients with neuroinflammation

$\dagger P<0.0001$ vs. normal patient control

$\ddagger P=0.001$ vs. patients with neuroinflammation

${ }^{\circ} P=0.06$ vs. patients with neuroinflammation 
CSF and serum. The detection limit was $31 \mathrm{ng} / \mathrm{L}$ for the NFL (NF-Light ${ }^{\circledR}$ Neurofilament ELISA kit) and $23.5 \mathrm{pg} /$ mL for NFH (Human Phosphorylated Neurofilament $\mathrm{H}$ ELISA kit). For samples below the detection limit, the values equal to limit of detection were used for the purpose of statistical analysis.

Concentrations of total tau protein, phosphorylated tau 181 and beta-amyloid 42 peptide were assayed using ELISA kits (INNOTEST ${ }^{\circledR}$ hTAU Ag, INNOTEST ${ }^{\circledR}$ PHOSPHO-TAU $_{(181 \mathrm{P})}$, INNOTEST $® \beta-$ AMYLOID $\left._{(1-42)}\right)$ from Innogenetics (now Fujirebio Europe, Ghent, Belgium). The detection limits declared by manufactures were $34 \mathrm{pg} / \mathrm{mL}$ for total tau protein, $13 \mathrm{pg} / \mathrm{mL}$ for phosphorylated tau 181 and $65 \mathrm{pg} / \mathrm{mL}$ for beta-amyloid 42 peptide. The analytical sensitivity of the used kits did not enable to measure concentrations of t-tau and p-tau proteins in serum.

Albumin was measured using immunonephelometry (IMMAGE, Beckman Coulter, Inc., Fullerton, CA).

\section{Calculations of ratios}

Albumin quotient $\left(\mathrm{Q}_{\text {albumin }}\right)$

A quotient between $\mathrm{CSF}$ and serum (CSF/serum) albumin concentrations $* 10^{-3}$ was used for evaluation of the blood-CSF barrier function.

\section{Indices for neurocytoskeletal proteins}

We investigated ratios between: 1) neurofilaments or tau proteins (CSF NFL/NFH, CSF p-tau/t-tau) and different neurofilaments with tau proteins (CSF NFL/t-tau, CSF NFL/p-tau, CSF NFH/t-tau, CSF NFH/p-tau) and 2) a neurocytoskeletal protein in CSF and the corresponding protein in serum (CSF/serum NFL, CSF/serum $\mathrm{NFH}$ ).

\section{Statistical analyses}

Since levels of the majority of evaluated variables were not normally distributed, differences among the four groups were analyzed using the Kruskal-Wallis test. When a significant difference among groups was shown, we performed pairwise comparison by the Mann-Whitney U tests ${ }^{20,35}$. These statistical analyses were performed using Statistica software, version 12.0 (StatSoft CR s.r.o. Prague, CR).

The diagnostic potential of the cytoskeletal proteins and calculated ratios were evaluated by calculating the areas under the curve (AUC) of the receiver operating characteristic (ROC) and by determination of the sensitivities and specificities using optimal cut-offs according to Youden index. These statistical calculations were performed by MedCalc statistical software (Ostend, Belgium).

The significance level for all tests was $P<0.05$.

\section{RESULTS}

Demographic and cognitive characteristics of the participants are summarized in Table 1. Age did not differ among groups. Females prevailed in the AD group which corresponds with epidemiological findings. The
MMSE score and ACE-R-CZ score were lower in AD and other dementias than in normal controls $(P \leq 0.0001$ for each comparison) and inflammatory patients (MMSE: $P<0.0001$ for each comparison, ACE-R-CZ: AD vs. IP $P=0.001$; OD vs. IP $P=0.06$ ).

$\mathrm{Q}_{\text {albumin }}$ was significantly higher in IP patients than in normal controls or the AD group $(P<0.005$ for each comparison).

\section{Neurocytoskeletal protein levels in CSF and serum}

The levels of NFL in CSF differ among the groups (Fig. 1A). A significant increase of CSF NFL levels was found in the OD group in comparison with the normal controls $(P=0.02)$. A comparison between dementia groups also showed higher levels in the OD group than in AD patients $(P=0.03)$. NFL levels in the serum were below the detection limit of the ELISA assay in 15 persons from all participants. Despite this fact, serum NFL levels were significantly elevated in OD patients compared with the AD group $(P=0.005)$ (Fig. 1B).

The changes in CSF NFH levels were similar to those observed in CSF NFL. The levels in the OD group were significantly elevated in comparison with normal controls and the $\mathrm{AD}$ group ( $P=0.02$ for either group) (Fig. 1C). Moreover, CSF NFH levels were also higher in IP patients than in $\mathrm{AD}$ patients $(P=0.02)$ and normal controls $(P=0.03)$. NFH levels in the serum did not significantly differ among groups (Fig. 1D). Nineteen values of serum NFH levels from all participants were lower than the detection limit of the ELISA kit.

The highest levels of t-tau in CSF were demonstrated in the AD group. A significant difference was observed between the AD group and the control group $(P=0.0002)$ or inflammatory patients $(P=0.0005)$ (Fig. $1 \mathrm{E})$. In addition, the CSF t-tau was significantly elevated in the OD group in comparison with the control group (OD vs. NC $P=0.004$ ) or inflammatory patients (OD vs. IP $P=0.008$ ).

Like t-tau, the highest CSF p-tau levels were also shown in AD patients (Fig. 1F). The increase of CSF p-tau in the AD group was significant when compared to levels in other groups (AD group vs. OD group $P=0.008$; AD group vs. NC group $P=0.003$; AD group vs. IP group $P=0.006)$. The CSF p-tau levels were similar in non-AD groups.

In the OD group, a female patient ( 71 years old) who had frontotemporal lobar degeneration in combination with amyotrophic lateral sclerosis was included. She had mildly impaired cognitive functions (MMSE score 25 points, ACE-R-CZ score 79 points). Despite the combination of two neurodegenerative processes, an extreme increase in neurocytoskeletal proteins was not observed in this patient. NFL and NFH levels in both serum and cerebrospinal fluid were within the range from the $25^{\text {th }}$ to the $75^{\text {th }}$ percentiles observed for the OD group (CSF NFL $6128 \mathrm{pg} / \mathrm{mL}$, serum NFL $958 \mathrm{pg} / \mathrm{mL}$, CSF NFH 1030 $\mathrm{pg} / \mathrm{mL}$ serum NFH $37.4 \mathrm{pg} / \mathrm{mL}$ ). The CSF t-tau (187 pg/ $\mathrm{mL})$ and p-tau (17 pg/mL) levels were lower, below $25^{\text {th }}$ percentile. Decreased tau levels influenced the calculated ratios of different neurofilaments with tau proteins, which were higher than the $75^{\text {th }}$ percentile of the OD group. 

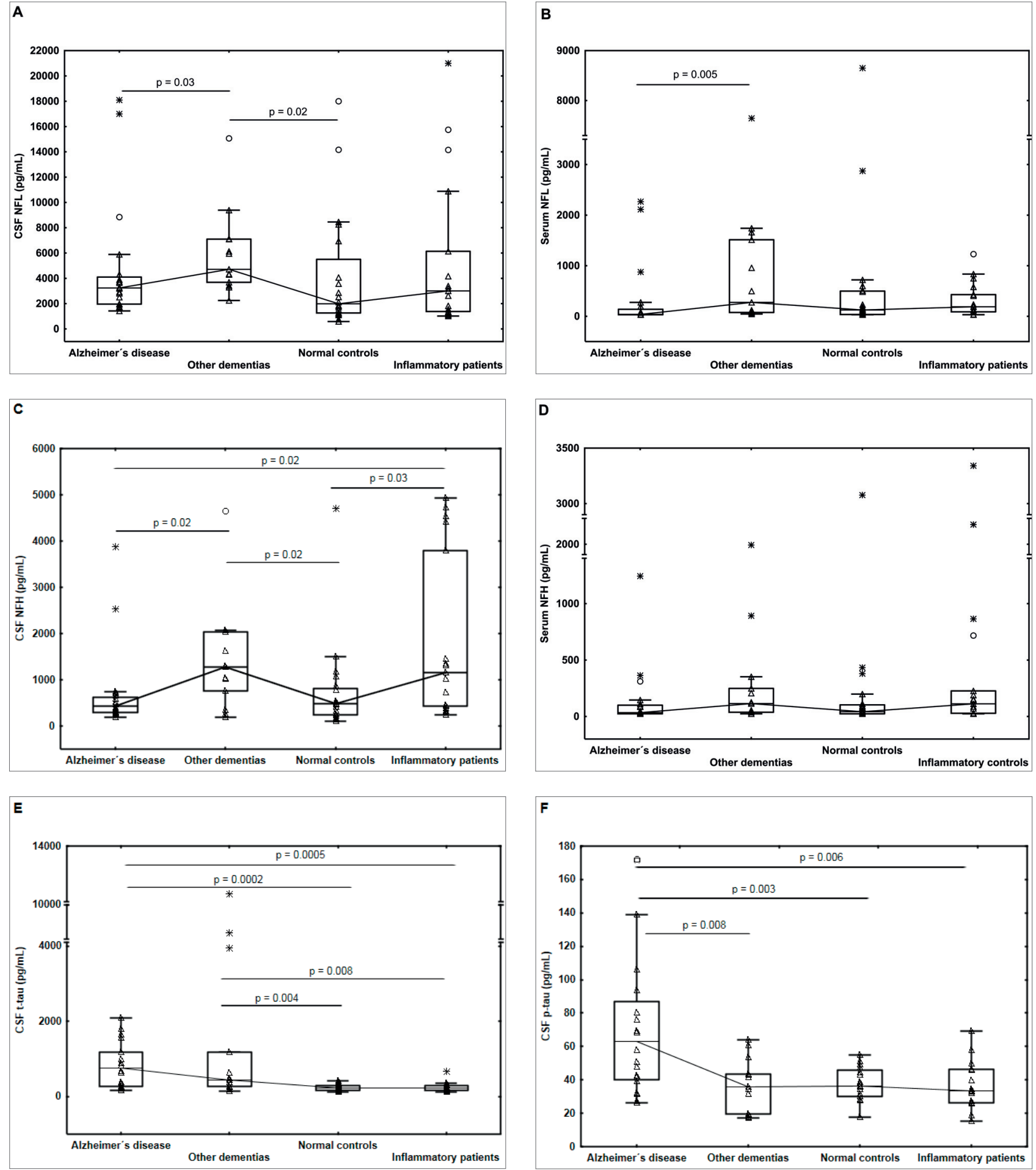

Fig. 1. Comparisons of levels among patients' groups (A) in neurofilament light subunits in cerebrospinal fluid; (B) in neurofilament light subunits in serum; (C) in neurofilament heavy subunits in cerebrospinal fluid; (D) in neurofilament heavy subunits in serum; (E) in total tau in cerebrospinal fluid; (F) in phosphorylated tau in cerebrospinal fluid. The box plots include the median (horizontal line - ) , values from the $25^{\text {th }}$ to the $75^{\text {th }}$ percentiles (boxes); bars (whiskers) above and below the box indicate the range of non-outliers. Non-outliers are indicated by triangles. Outliers and extremes are shown by circles and asterisks (the outlier values are outside the 1.5 box length from the upper and lower value of the box; the extreme values are outside the 3 box length from the upper and lower value of the box).

CSF - cerebrospinal fluid; NFH - neurofilament heavy subunits; NFL - neurofilament light subunits; p-tau - phosphorylated tau; t-tau - total tau. 

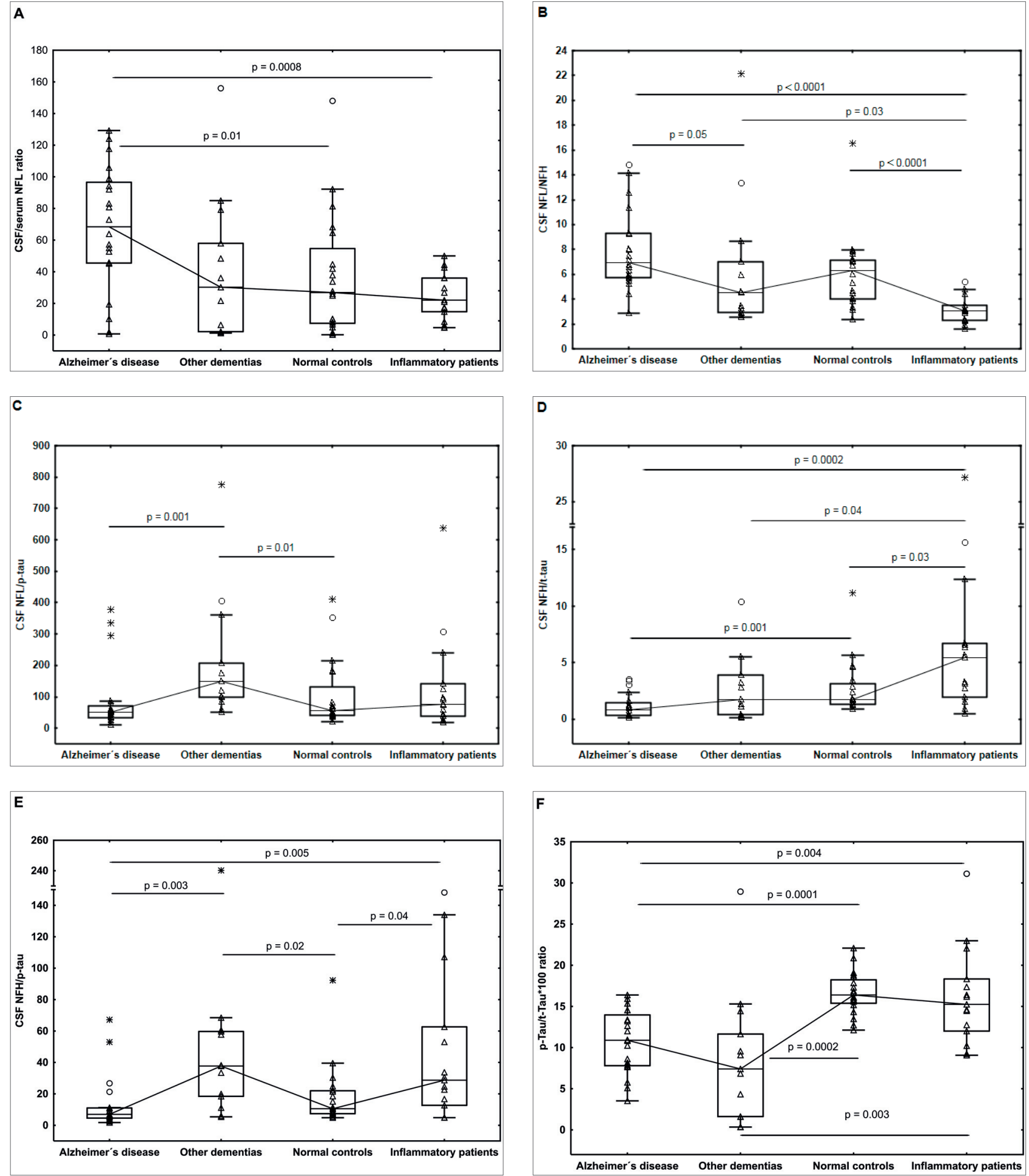

Fig. 2. Ratio comparisons among patients' groups (A) CSF/serum NFL ratio; (B) CSF NFL/NFH ratio; (C) CSF NFL/p-tau ratio; (D) CSF NFH/t-tau; (E) CSF NFH/p-tau; (F) CSF p-tau/t-tau

The ratios CSF/serum NFH and CSF NFL/t-tau, which did not differ among groups, are not shown.

The box plots include median (horizontal line - ), values from the $25^{\text {th }}$ to the $75^{\text {th }}$ percentiles (boxes); bars (whiskers) above and below the box indicate the range of non-outliers. Non-outliers are indicated by triangles. Outliers and extremes are shown by circles and asterisks (the outlier values are outside the 1.5 box length from the upper and lower value of the box; the extreme values are outside the 3 box length from the upper and lower value of the box).

CSF - cerebrospinal fluid; NFH - neurofilament heavy subunits; NFL - neurofilament light subunits; p-tau - phosphorylated tau; t-tau - total tau 
Table 2. Comparison of areas under the curve of receiver operating characteristics of neurocytoskeletal proteins and their ratios among patients' groups. Cytoskeletal proteins and ratios with AUC $>0.70$ are shown.

\begin{tabular}{|c|c|c|c|c|}
\hline & $\operatorname{AUC}(95 \% \mathrm{Cl})$ & Cut-off & $\operatorname{Sn}(\%)$ & $\mathrm{Sp}(\%)$ \\
\hline \multicolumn{5}{|c|}{ Normal controls vs. Alzheimer's disease } \\
\hline \multicolumn{5}{|l|}{ Cytoskeletal proteins } \\
\hline CSF t-tau $(\mathrm{pg} / \mathrm{mL})$ & $0.84(0.70$ to 0.94$) \dagger$ & $>371$ & 65 & 95 \\
\hline CSF p-tau $(\mathrm{pg} / \mathrm{mL})$ & $0.78(0.62$ to 0.90$)$ & $>55$ & 55 & 100 \\
\hline \multicolumn{5}{|l|}{ Ratios } \\
\hline CSF p-tau/t-tau & $0.91(0.78$ to 0.98$) \dagger$ & $\leq 0.13$ & 75 & 90 \\
\hline CSF NFH/t-tau & $0.80(0.64$ to 0.91$)$ & $\leq 1.2$ & 75 & 80 \\
\hline CSF NFL/t-tau & $0.75(0.59$ to 0.87$)$ & $\leq 6$ & 55 & 90 \\
\hline \multicolumn{5}{|c|}{ Normal controls vs. other dementias } \\
\hline \multicolumn{5}{|l|}{ Cytoskeletal proteins } \\
\hline CSF t-tau (pg/mL) & $0.80(0.63$ to 0.92$) \ddagger$ & $>421$ & 53 & 100 \\
\hline CSF NFL (pg/mL) & $0.75(0.57$ to 0.88$)$ & $>2859$ & 92 & 65 \\
\hline CSF NFH (pg/mL) & $0.75(0.57$ to 0.88$)$ & $>855$ & 69 & 80 \\
\hline \multicolumn{5}{|l|}{ Ratios } \\
\hline CSF p-tau/t-tau & $0.89(0.73$ to 0.97$) \ddagger$ & $\leq 0.12$ & 77 & 100 \\
\hline CSF NFL/p-tau & $0.77(0.59$ to 0.90$)$ & $>82$ & 85 & 75 \\
\hline CSF NFH/p-tau & $0.74(0.73$ to 0.97$)$ & $>30$ & 62 & 90 \\
\hline \multicolumn{5}{|c|}{ Alzheimer disease vs. other dementias } \\
\hline \multicolumn{5}{|l|}{ Cytoskeletal proteins } \\
\hline Serum NFL (pg/mL) & $0.79(0.62$ to 0.91$)+\dagger$ & $\leq 38.4$ & 60 & 100 \\
\hline CSF p-tau $(\mathrm{pg} / \mathrm{mL})$ & $0.78(0.60$ to 0.90$)$ & $>64$ & 50 & 100 \\
\hline CSF NFH (pg/mL) & $0.74(0.56$ to 0.88$)$ & $\leq 741$ & 90 & 77 \\
\hline \multicolumn{5}{|l|}{ Ratios } \\
\hline CSF NFL/p-tau & $0.84(0.67$ to 0.94$) \dagger \dagger$ & $\leq 79$ & 80 & 85 \\
\hline CSF NFH/p-tau & $0.82(0.64$ to 0.93$)$ & $\leq 11$ & 75 & 85 \\
\hline CSF NFL/NFH & $0.71(0.52$ to 0.85$)$ & $>6$ & 70 & 69 \\
\hline \multicolumn{5}{|c|}{ Normal controls vs. inflammatory patients } \\
\hline \multicolumn{5}{|l|}{ Cytoskeletal proteins } \\
\hline CSF NFH (pg/mL) & $0.71(0.54 \text { to } 0.85)^{*}$ & $>855$ & 59 & 80 \\
\hline \multicolumn{5}{|l|}{ Ratios } \\
\hline CSF NFL/NFH & $0.89(0.74 \text { to } 0.97)^{*}$ & $\leq 3.5$ & 82 & 85 \\
\hline CSF NFH/t-tau & $0.72(0.54$ to 0.86$)$ & $>4.7$ & 53 & 90 \\
\hline \multicolumn{5}{|c|}{ Alzheimer disease vs. inflammatory patients } \\
\hline \multicolumn{5}{|l|}{ Cytoskeletal proteins } \\
\hline CSF t-tau (pg/mL) & $0.85(0.69 \text { to } 0.95)^{* *}$ & $>356$ & 65 & 93 \\
\hline CSF p-tau (pg/mL) & $0.78(0.610$ to 0.90$)$ & $>49.5$ & 60 & 87 \\
\hline \multicolumn{5}{|l|}{ Ratios } \\
\hline CSF NFL/NFH & $0.96(0.83 \text { to } 1.0)^{* *}$ & $>5.4$ & 85 & 100 \\
\hline CSF NFH/t-tau & $0.87(0.71$ to 0.96$)$ & $\leq 1.2$ & 75 & 87 \\
\hline CSF / serum NFL & $0.83(0.67$ to 0.94$)$ & $>44.4$ & 80 & 94 \\
\hline
\end{tabular}

AUC - area under the curve; CSF - cerebrospinal fluid; Cl - confidence interval; NFL - neurofilament light subunits; NFH - neurofilament heavy subunits; Sn - sensitivity; Sp - specificity; tau - total tau; p-tau - phosphorylated tau; vs. - versus

$\dagger+$ AUC were not significantly different between the CSF p-tau/t-tau ratio and CSF t-tau levels when normal controls vs. Alzheimer’s disease or other dementias were compared.

$\dagger \uparrow$ AUC were not significantly different between the NFL/p-tau ratio and serum NFL levels when Alzheimer's disease vs. other dementias were compared.

*AUC were significantly different between the CSF NFL/NFH ratio and CSF NFH levels $(P=0.043)$. AUC of CSF NFL/NFH and CSF NFH/ttau ratios were not significantly different among themselves when normal controls vs. inflammatory patients were compared.

**The difference between the CSF NFL/NFH ratio and CSF t-tau levels was not significant but the $P$ value was on the borderline of significance ( $P=0.054)$. 
Ratios of different neurocytoskeletal proteins in CSF and serum

The CSF/serum NFL ratio was significantly higher in the AD group than that in normal controls $(P=0.01)$ or in the IP group $(P=0.0008)$ (Fig. $2 \mathrm{~A})$. The non-AD groups did not differ among themselves. The CSF/serum NFH ratio was similar in all groups.

\section{CSF ratios of individual neurocytoskeletal proteins}

The lowest values of the CSF NFL/NFH ratio were seen in the IP group and they significantly differed from those in normal controls $(P<0.0001)$ and any dementia patients (IP vs. AD $P<0.0001$; IP vs. OD $P=0.03$ ). Additionally, the CSF NFL/NFH ratio was significantly lower in the OD group than in the AD group $(P=0.05)$ (Fig. 2B).

The CSF NFL/t-tau ratio CSF did not show any differences among groups, while the CSF NFL/p-tau ratio was significantly higher in the OD group than that in the AD group $(P=0.001)$ or the $\mathrm{NC}$ group $(P=0.01)$ (Fig. $2 \mathrm{C})$.

The ratios of CSF NFH/t-tau or CSF NFH/p-tau differed significantly among groups (Fig. 2D and 2E). Both ratios $\mathrm{CSF} \mathrm{NFH} / \mathrm{t}$-tau and $\mathrm{CSF} \mathrm{NFH} / \mathrm{p}$-tau showed the lowest values in the $\mathrm{AD}$ group. The $\mathrm{CSF} \mathrm{NFH} / \mathrm{t}$-tau ratio was lower in $\mathrm{AD}$ patients than in the control group $(P=0.001)$ or the IP group $(P=0.0002)$. Moreover, the CSF $\mathrm{NFH} / \mathrm{t}$-tau ratio was lower in the OD group or normal controls than that in the IP group (OD vs. IP $P=0.04$; NC vs. IP $P=0.03)$. The $\mathrm{CSF} N F H / p$-tau ratio significantly differed between the AD group and the OD group $(P=0.003)$ or the IP group $(P=0.005)$ and between normal controls and the OD group $(P=0.02)$ or the IP group $(P=0.04)$.

Prominent differences were observed in the ratio CSF p-tau/t-tau (Fig. 2F). The ratio was significantly lower in the AD group and the OD group than that in normal controls (AD group vs. NC group $P=0.0001$; OD group vs. NC group $P=0.0002)$ and similarly the ratio values were also significantly lower in patients of any dementia group than in the group of patients with inflammatory diseases (AD group vs. IP group $P=0.004$; OD group vs. IP group $P=0.003$ ).

Altogether, 1) the CSF NFL/NFH ratio was the best at differentiating between patients with aseptic neuro-infections and the AD group or normal controls; 2) the ratio CSF p-tau/t-tau showed the highest difference between normal controls and AD patients or other dementias, and 3 ) the CSF NFL/p-tau and CSF NFH/p-tau ratios differentiated between $\mathrm{AD}$ patients and patients with other dementias very well.

\section{Evaluation of neurocytoskeletal proteins and their ratios according to the area under the ROC curves}

The CSF NFL/NFH ratio showed the highest discrimination potency for differentiation between the inflammatory group and AD or NC groups evaluated by the area under the ROC curves. The optimal results for discrimination between the NC group and the AD or OD groups were observed for the CSF p-tau/t-tau ratio and discrimination between the $\mathrm{OD}$ and $\mathrm{AD}$ groups were the best using CSF NFL/p-tau, CSF NFH/p-tau and serum
NFL. The AUC for the CSF p-tau/t-tau, CSF NFL/p-tau and $\mathrm{CSF} N F H / p$-tau ratios with the highest values did not significantly differ from those of the individual proteins, while the AUC for the CSF NFL/NFH ratio was significantly higher than that of the individual proteins with the highest values of AUC (Table 2).

\section{DISCUSSION}

In this study we determined neurofilaments NFL and $\mathrm{NFH}$ together with CSF tau proteins in patients with AD, other dementias and aseptic inflammatory neuro-infections. This allowed us to evaluate the clinical relevance of various ratios based on the neuron-specific proteins. We demonstrated that CSF NFH levels differed between inflammatory patients and normal controls or AD patients. Unfortunately, levels of individual NFL and NFH proteins either in CSF or in serum did not seem to differentiate between cognitively normal subjects and patients with Alzheimer's disease. We observed that the CSF NFL/ $\mathrm{NFH}$ ratio was significantly elevated in the AD group compared to patients with inflammatory diseases or other dementias and the CSF p-tau/t-tau ratio showed the best values for discrimination between cognitively normal controls and $\mathrm{AD}$ or other dementias. The findings of the highest elevation of CSF t-tau and CSF p-tau in AD patients were the expected results ${ }^{9}$.

Some ratios of neuron-specific proteins might contribute to differentiation between patients with dementias and normal controls. The CSF p-tau/t-tau ratio significantly decreased in both groups of dementias. The most prominent reduction was shown in other dementias but not in AD in which the marked elevation of $\mathrm{p}$-tau caused the lesser decrease of the CSF p-tau/t-tau ratio. It seems that the elevation of CSF t-tau levels considered as a biomarker of cortical axonal pathology ${ }^{10}$ mostly contributed to the decrease of the CSF p-tau/t-tau ratio in various types of dementias. Our results are in good agreement with findings of a recent study of Pijnenburg et al. ${ }^{20}$. The authors identified the decreased CSF p-tau/t-tau ratio as a biomarker for one of the subtypes of frontotemporal dementia (FTD) even with prognostic potential although a similar decrease was not observed in the non-differentiated group of FTD in their previous study ${ }^{18}$. Changes in ratio between CSF p-tau and CSF t-tau were also reported in other neurodegenerative diseases such as CreutzfeldtJacob disease and amyotrophic lateral sclerosis ${ }^{36,37}$.

Alterations in neurofilaments have been observed in multiple neurological diseases including AD (ref., ${ }^{5,38}$ ). Specific subclasses of neurons varying in the NF content in different regions of the central nervous system have been described ${ }^{44}$. It is characteristic for AD that neurons containing neurofilament proteins in regions of neocortex as well as medial temporal lobe structures have a greater predisposition to form NFT and degenerate ${ }^{2,45}$. In the opposite case, neurons lacking neurofilaments are not susceptible to these pathological changes ${ }^{46}$. We were unable to confirm observations of some studies reporting increased levels of CSF NFL or CSF NFH in the AD 
group $^{5,9}$. Based on the metaanalysis ${ }^{9}$, we expected higher levels of CSF NFL in the AD group. However, not all studies report a significant increase in neurofilament levels. In a recent study, levels of NF in CSF as well as in serum were significantly elevated in the Guillain-Barré syndrome or amyotrophic lateral sclerosis, but not in AD (ref. $\left.{ }^{13}\right)$. Similarly, a study primarily focused on the changes of various markers including neurofilaments over time did not mention the difference in baseline NF levels between the AD group and controls ${ }^{47}$. In our study we observed the highest CSF and serum NFL levels in the group of other dementias. The median of NFL levels in this group was 2.37 times for CSF and 2.2 times for serum higher in comparison with normal control, while the CSF elevation in the AD group was only non-significantly higher, similarly as in the group of patients with inflammatory diseases. Our observation of increased CSF NFL levels in patients with other dementias is consistent with previous studies ${ }^{5}$. The highest levels of CSF NFL were found in frontotemporal dementia, vascular dementia and mixed AD in comparison with other types of dementia ${ }^{19}$.

Serum levels of NFL and NFH did not differentiate normal controls and dementia groups. The use of highly sensitive immunoanalytical methods that allow us to quantify very low levels of neurofilaments in serum/plasma can increase their potential as biomarkers for dementias ${ }^{48}$. The most recent studies using single molecule array (Simoa) technique for NFL determination have shown significantly higher serum NFL levels in the AD group even in prodromal stadium compared to normal controls. However the overlap between the AD and other diagnostic groups was obvious $^{14,15}$. Despite this limitation plasma NFL levels may be a useful noninvasive biomarker of neurodegenerative processes ${ }^{15}$.

Considering the different functions of individual NF chains and that variations in NF stoichiometry are assumed to be involved in the process of neurodegeneration $^{49}$, we were interested whether the NFL/NFH ratio has any diagnostic potential. The changes in the values of the CSF NFL/NFH ratio may reflect the alteration in the NF stoichiometry in certain neurological diseases. Unfortunately, the ratio of the CSF NLF/NFH ratio did not support the diagnosis in either AD or other dementias. However, the neuron damage in neurodegeneration and neuroinflammation in aseptic neuro-infections was reflected in the NF ratio in different ways. The expression of NF levels in the CSF NFL/NFH ratio distinguished between patients with inflammatory diseases and the normal controls or AD with great efficiency.

The ratio of NFL as a biomarker of white matter axonal damage and tau were suggested to be useful for distinguishing between early-onset $\mathrm{AD}$ and frontotemporal dementia $^{21}$. Both CSF NFL/p-tau and CSF NFH/p-tau ratios were significantly reduced in $\mathrm{AD}$ compared to other dementias. The decrease of the ratio in the AD group reflected both lower levels of neurofilaments and higher levels of p-tau in AD patients than in patients with other dementias.

\section{CONCLUSION}

In conclusion, we demonstrated a different impact of axonal impairment in the neurodegeneration and neuroinflammation in aseptic neuro-infections to the CSF and serum levels of neuronal cytoskeletal proteins. While the tau proteins as well as their index differentiated between $\mathrm{AD}$ or OD patients and cognitively normal subjects, CSF levels of neurofilaments expressed as their index might contribute to the discrimination between patients with neuroinflammation and normal controls or AD patients.

Acknowledgement: The study was supported by Research Project Charles University in Prague PROGRES Q25 and Q35, by the project of National Institute of Mental Health NPU I (LO1611) and by Ministry of Health, Czech Republic - conceptual development of research organization RVO VFN 64165, General University Hospital in Prague, Czech Republic.

We wish to thank Dr. Benáková for the albumin measurement of all of our specimens.

Author contributions: LF: study design, data analysis and interpretation, writing of the manuscript; AB: study design, collection and analysis of clinical data, clinical evaluation, general corrections; JS: laboratory analysis, data analysis.

Conflict of interest statement: The authors state that there are no conflicts of interest regarding the publication of this article.

\section{REFERENCES}

1. Perrot R, Eyer J. Neuronal intermediate filaments and neurodegenerative disorders. Brain Res Bull 2009;80:282-95.

2. Vickers JC, Dickson TC, Adlard PA, Saunders HL, King CE, McCormack $\mathrm{G}$. The cause of neuronal degeneration in Alzheimer's disease. Prog Neurobiol 2000;60:139-65.

3. Kolarova M, Garcia-Sierra F, Bartos A, Ricny J, Ripova D. Structure and pathology of tau protein in Alzheimer disease. Int J Alzheimers Dis 2012;2012:731526.

4. Zetterberg H. Cerebrospinal fluid biomarkers for Alzheimer's disease: current limitations and recent developments. Curr Opin Psychiatry 2015;28:402-9.

5. Petzold A, Keir G, Warren J, Fox N, Rossor MN. A systematic review and meta-analysis of CSF neurofilament protein levels as biomarkers in dementia. Neurodegener Dis 2007;4:185-94.

6. Hort J, Bartos A, Pirttila T, Scheltens P. Use of cerebrospinal fluid biomarkers in diagnosis of dementia across Europe. Eur J Neurol 2010;17:90-6.

7. van Harten AC, Kester MI, Visser PJ, Blankenstein MA, Pijnenburg YA, van der Flier WM, Scheltens P. Tau and $p$-tau as CSF biomarkers in dementia: a meta-analysis. Clin Chem Lab Med 2011;49:353-66.

8. Llorens F, Kruse N, Schmitz M, Shafiq M, da Cunha JE, Gotzman N, Zafar S, Thune K, de Oliveira JR, Mollenhauer B, Zerr I. Quantification of CSF biomarkers using an electrochemiluminescence-based detection system in the differential diagnosis of $A D$ and SCJD. J Neurol 2015;262:2305-11.

9. Olsson B, Lautner R, Andreasson U, Ohrfelt A, Portelius E, Bjerke M, Holtta M, Rosen C, Olsson C, Strobel G, Wu E, Dakin K, Petzold M, Blennow K, Zetterberg H. CSF and blood biomarkers for the diagnosis of Alzheimer's disease: a systematic review and meta-analysis. Lancet Neurol 2016;15:673-84.

10. Mattsson N. CSF biomarkers in neurodegenerative diseases. Clin Chem Lab Med 2011;49:345-52.

11. Rudrabhatla P, Jaffe H, Pant HC. Direct evidence of phosphory- 
lated neuronal intermediate filament proteins in neurofibrillary tangles (NFTs): phosphoproteomics of Alzheimer's NFTs. FASEB J 2011;25:3896-905.

12. Zetterberg H, Skillback T, Mattsson N, Trojanowski JQ, Portelius E, Shaw LM, Weiner MW, Blennow K, Alzheimer's Disease Neuroimaging Initiative. Association of Cerebrospinal Fluid Neurofilament Light Concentration With Alzheimer Disease Progression. JAMA Neurol 2016:73:60-7.

13. Gaiottino J, Norgren N, Dobson R, Topping J, Nissim A, Malaspina A, Bestwick JP, Monsch AU, Regeniter A, Lindberg RL, Kappos L, Leppert D, Petzold A, Giovannoni G, Kuhle J. Increased neurofilament light chain blood levels in neurodegenerative neurological diseases. PLoS One 2013;8:e75091.

14. Zhou W, Zhang J, Ye F, Xu G, Su H, Su Y, Zhang X, Alzheimer's Disease Neuroimaging Initiative. Plasma neurofilament light chain levels in Alzheimer's disease. Neurosci Lett 2017;650:60-4.

15. Mattsson N, Andreasson U, Zetterberg H, Blennow K, Alzheimer's Disease Neuroimaging I. Association of Plasma Neurofilament Light With Neurodegeneration in Patients With Alzheimer Disease. JAMA Neurol 2017;74:557-66.

16. Brettschneider J, Petzold A, Schottle D, Claus A, Riepe M, Tumani $\mathrm{H}$. The neurofilament heavy chain $(\mathrm{NfH})$ in the cerebrospinal fluid diagnosis of Alzheimer's disease. Dement Geriatr Cogn Disord 2006;21:291-5.

17. de Jong D, Jansen RW, Pijnenburg YA, van Geel WJ, Borm GF, Kremer $\mathrm{HP}$, Verbeek MM. CSF neurofilament proteins in the differential diagnosis of dementia. J Neurol Neurosurg Psychiatry 2007;78:936-8.

18. Pijnenburg YA, Janssen JC, Schoonenboom NS, Petzold A, Mulder C, Stigbrand T, Norgren N, Heijst H, Hack CE, Scheltens P, Teunissen CE. CSF neurofilaments in frontotemporal dementia compared with early onset Alzheimer's disease and controls. Dement Geriatr Cogn Disord 2007;23:225-30.

19. Skillback T, Farahmand B, Bartlett JW, Rosen C, Mattsson N, Nagga K, Kilander L, Religa D, Wimo A, Winblad B, Rosengren L, Schott JM Blennow K, Eriksdotter M, Zetterberg H. CSF neurofilament light differs in neurodegenerative diseases and predicts severity and survival. Neurology 2014;83:1945-53.

20. Pijnenburg YA, Verwey NA, van der Flier WM, Scheltens P, Teunissen CE. Discriminative and prognostic potential of cerebrospinal fluid phosphoTau/tau ratio and neurofilaments for frontotemporal dementia subtypes. Alzheimers Dement (Amst) 2015;1:505-12.

21. Sjogren $M$, Rosengren $L$, Minthon $L$, Davidsson $P$, Blennow $K$, Wallin A. Cytoskeleton proteins in CSF distinguish frontotemporal dementia from AD. Neurology 2000;54:1960-4.

22. Mioshi E, Dawson K, Mitchell J, Arnold R, Hodges JR. The Addenbrooke's Cognitive Examination Revised (ACE-R): a brief cog nitive test battery for dementia screening. Int J Geriatr Psychiatry 2006;21:1078-85.

23. Hummelová-Fanfrdlová Z, Rektorová I, Sheardová K, Bartoš A, Línek V, Ressner P, Zapletalová J, Vyhnálek M, Hort J. Czech adaptation of Addenbrooke's Cognitive Examination. Ceskoslovenska Psychologie 2009;53:376-89.

24. Bartoš A, Raisová M. The Mini-Mental State Examination (MMSE) - Czech norms and cut-offs for mild dementia and mild cognitive impairment due to Alzheimer disease. Dement and Geriatr Cogn Disord 2016;42:50-7.

25. Bartoš $A$, Raisová $M$, Kopeček $M$. Amendment of the Czech Addenbrooke's cognitive examination (ACE-CZ). Cesk Slov Neurol N 2011;74:681-4

26. Bartoš $A$, Raisová $M$, Kopeček $M$. The reasons and the process of amendment of the Czech Addenbrooke's cognitive examination (ACE-CZ). Cesk Slov Neurol N 2011;74:e1-e6.

27. Folstein MF, Folstein SE, McHugh PR. "Mini-mental state". A practica method for grading the cognitive state of patients for the clinician. J Psychiatr Res 1975;12:189-98.

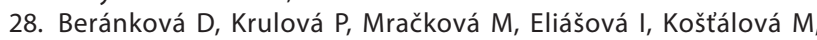
Janoušová $\mathrm{E}$, Stehnová I, Bar $\mathrm{M}$, Ressner $\mathrm{P}$, Nilius $\mathrm{P}$, Tomagová $\mathrm{M}$, Rektorová I. Addenbrooke's Cognitive Examination - Approximate Normal Values for the Czech Population (in Czech). Cesk Slov Neuro N 2015;78/111:300-5.

29. Scheltens $P$, Leys $D$, Barkhof $F$, Huglo $D$, Weinstein HC, Vermersch $P$, Kuiper M, Steinling M, Wolters EC, Valk J. Atrophy of medial temporal lobes on MRI in "probable" Alzheimer's disease and normal ageing: diagnostic value and neuropsychological correlates. J Neuro Neurosurg Psychiatry 1992;55:967-72.
30. Bartoš A, Zach P, Diblíková F, Tintěra J, Řípová D, Brunovský M. Visual rating of medial temporal lobe atrophy on magnetic resonance imaging in Alzheimer's disease. Psychiatrie 2007;11:49-52.

31. McKhann GM, Knopman DS, Chertkow H, Hyman BT, Jack CR, Jr., Kawas CH, Klunk WE, Koroshetz WJ, Manly JJ, Mayeux R, Mohs RC, Morris JC, Rossor MN, Scheltens P, Carrillo MC, Thies B, Weintraub S, Phelps $\mathrm{CH}$. The diagnosis of dementia due to Alzheimer's disease: recommendations from the National Institute on Aging-Alzheimer's Association workgroups on diagnostic guidelines for Alzheimer's disease. Alzheimers Dement 2011;7:263-9.

32. Bartoš A, Čechová L, Švarcová J, Říčný J, Řípová D. Cerebrospinal fluid triplet (tau proteins and beta-amyloid) in the diagnosis of Alzheimer-Fischer disease [Likvorový triplet (tau proteiny a betaamyloid) v diagnostice Alzheimerovy-Fischerovy nemoci]. Cesk Slov Neurol N 2012;75/108:587-94.

33. Deisenhammer F, Egg R, Giovannoni G, Hemmer B, Petzold A Sellebjerg F, Teunissen C, Tumani H. EFNS guidelines on diseasespecific CSF investigations. Eur J Neurol 2009;16:760-70.

34. Teunissen CE, Petzold A, Bennett JL, Berven FS, Brundin L, Comabella M, Franciotta D, Frederiksen JL, Fleming JO, Furlan R, Hintzen RQ, Hughes SG, Johnson MH, Krasulova E, Kuhle J, Magnone MC, Rajda C, Rejdak K, Schmidt HK, van Pesch V, Waubant E, Wolf C, Giovannoni G, Hemmer B, Tumani H, Deisenhammer F. A consensus protocol for the standardization of cerebrospinal fluid collection and biobanking. Neurology 2009;73:1914-22.

35. Bjerke M, Andreasson U, Rolstad S, Nordlund A, Lind K, Zetterberg H, Edman A, Blennow K, Wallin A. Subcortical vascular dementia biomarker pattern in mild cognitive impairment. Dement Geriatr Cogn Disord 2009;28:348-56.

36. Skillback T, Rosen C, Asztely F, Mattsson N, Blennow K, Zetterberg $H$. Diagnostic performance of cerebrospinal fluid total tau and phosphorylated tau in Creutzfeldt-Jakob disease: results from the Swedish Mortality Registry. JAMA Neurol 2014;71:476-83.

37. Grossman M, Elman L, McCluskey L, McMillan CT, Boller A, Powers J, Rascovsky K, Hu W, Shaw L, Irwin DJ, Lee VM, Trojanowski JQ. Phosphorylated tau as a candidate biomarker for amyotrophic lateral sclerosis. JAMA Neurol 2014;71:442-8.

38. Liu Q, Xie F, Alvarado-Diaz A, Smith MA, Moreira PI, Zhu X, Perry G. Neurofilamentopathy in neurodegenerative diseases. Open Neurol J 2011;5:58-62.

39. Fialova L, Bartos A, Svarcova J, Zimova D, Kotoucova J, Malbohan I. Serum and cerebrospinal fluid light neurofilaments and antibodies against them in clinically isolated syndrome and multiple sclerosis. J Neuroimmunol 2013;262:113-20.

40. Rejdak K, Kuhle J, Ruegg S, Lindberg RL, Petzold A, Sulejczak D, Papuc E, Rejdak R, Stelmasiak Z, Grieb P. Neurofilament heavy chain and heat shock protein 70 as markers of seizure-related brain injury. Epilepsia 2012;53:922-7.

41. Dujmovic I. Cerebrospinal fluid and blood biomarkers of neuroaxonal damage in multiple sclerosis. Mult Scler Int 2011;2011:767083.

42. Kaiserova M, Grambalova Z, Otruba P, Stejskal D, Prikrylova Vranova $\mathrm{H}$, Mares J, Mensikova K, Kanovsky P. Cerebrospinal fluid levels of chromogranin A and phosphorylated neurofilament heavy chain are elevated in amyotrophic lateral sclerosis. Acta Neurol Scand 2017 Feb 10. doi: 10.1111/ane.12735. [Epub ahead of print]

43. Studahl M, Rosengren L, Gunther G, Hagberg L. Difference in pathogenesis between herpes simplex virus type 1 encephalitis and tickborne encephalitis demonstrated by means of cerebrospinal fluid markers of glial and neuronal destruction. J Neurol 2000;247:636-42.

44. Vickers JC, Costa M. The neurofilament triplet is present in distinct subpopulations of neurons in the central nervous system of the guinea-pig. Neuroscience 1992;49:73-100.

45. Vickers JC, Riederer BM, Marugg RA, Buee-Scherrer V, Buee L, Delacourte A, Morrison JH. Alterations in neurofilament protein immunoreactivity in human hippocampal neurons related to normal aging and Alzheimer's disease. Neuroscience 1994;62:1-13.

46. Vickers JC, Mitew S, Woodhouse A, Fernandez-Martos CM, Kirkcaldie MT, Canty AJ, McCormack GH, King AE. Defining the earliest pathological changes of Alzheimer's disease. Curr Alzheimer Res 2016;13:281-7.

47. Kester MI, Scheffer PG, Koel-Simmelink MJ, Twaalfhoven $H$, Verwey NA, Veerhuis R, Twisk JW, Bouwman FH, Blankenstein MA, Scheltens $P$, Teunissen C, van der Flier WM. Serial CSF sampling in Alzheimer's disease: specific versus non-specific markers. Neurobiol Aging 2012;33:1591-8. 
48. Kuhle J, Barro C, Andreasson U, Derfuss T, Lindberg R, Sandelius A, Liman V, Norgren N, Blennow K, Zetterberg H. Comparison of three analytical platforms for quantification of the neurofilament light chain in blood samples: ELISA, electrochemiluminescence immunoassay and Simoa. Clin Chem Lab Med 2016;54:1655-61.
49. Szaro BG, Strong MJ. Post-transcriptional control of neurofilaments: New roles in development, regeneration and neurodegenerative disease. Trends Neurosci 2010;33:27-37. 